\title{
Identification of proteins expressed by Babesia bigemina kinetes
}

\author{
Gamila A. R. Bohaliga', Wendell C. Johnson², Naomi S. Taus ${ }^{1,2}$, Hala E. Hussein ${ }^{1,3}$, Reginaldo G. Bastos ${ }^{1}$, \\ Carlos E. Suarez ${ }^{1,2}$, Glen A. Scoles ${ }^{1,2}$ and Massaro W. Ueti ${ }^{1,2,4^{*}}$
}

\begin{abstract}
Background: Babesia bigemina is an apicomplexan parasite transovarially transmitted via Rhipicephalus ticks that infect red blood cells and causes bovine babesiosis, a poorly controlled severe acute disease in cattle. New methods of control are urgently needed, including the development of transmission blocking vaccines (TBV). Babesia bigemina reproduces sexually in the gut of adult female $R$. microplus upon acquisition following a blood meal. Sexual reproduction results in zygotes that infect gut epithelial cells to transform into kinete stage parasites, which invade tick ovaries and infects the egg mass. The subsequent tick generation transmits B. bigemina upon feeding on bovine hosts. An important limitation for developing novel TBV is that the pattern of protein expression in B. bigemina tick stages, such as the kinete stage, remain essentially uncharacterized.
\end{abstract}

Results: We determined the protein expression profile of three B. bigemina putative tick stage candidates BbiKSP (BBBOND_0206730), CCp2 and CCP3. We found that BbiKSP expression was restricted to B. bigemina kinetes. CCp2 and CCp3, previously shown to be expressed by induced sexual stages, were also expressed by kinetes. Importantly, none of these proteins were expressed by B. bigemina blood stages.

Conclusions: Babesia bigemina kinetes express BbiKSP, CCp2 and CCp3 proteins, therefore, these proteins may play important roles during $B$. bigemina development within tick hemolymph and may serve as potential candidate targets for the development of TBV.

Keywords: Babesia bigemina, Kinete stage-specific protein, in vitro induced sexual stages, Kinete, Rhipicephalus microplus

\section{Background}

The apicomplexan parasite Babesia bigemina is an etiological agent of bovine babesiosis, a disease responsible for significant economic losses for the cattle industry. Bovine babesiosis is distributed globally and is transmitted transovarially by multiple Ixodidae tick species such as Rhipicephalus microplus, the major vector for B. bigemina. This parasite has a complex life-cycle that includes asexual reproduction while developing in bovine host erythrocytes and sexual reproduction in the midgut of the tick vector $[1,2]$. The disease is poorly controlled and new vaccines are urgently needed. Ideally vaccines

\footnotetext{
*Correspondence: massaro ueti@wsu.edu

${ }^{2}$ Animal Disease Research Unit, USDA-ARS, Pullman, WA 99164-6630, USA

Full list of author information is available at the end of the article
}

should be targeted at controlling both the development of debilitating or lethal acute disease (blood-stage vaccines), and transmission by ticks (transmission-blocking vaccines $[\mathrm{TBV}])$. However, vaccine development is currently limited by our poor understanding of parasite biology, especially at the molecular level. This study is focused on defining proteins that are exclusively expressed in tick stages as candidates for future TBV development against B. bigemina. To produce effective strategies to block tick infection and parasite transmission, a comprehensive understanding of the parasite's development in the tick vector is required.

The annotated genomes for several related arthropodborne apicomplexan parasites such as Plasmodium falciparum, P. vivax, B. bovis, B. bigemina, B. ovata and B. sp. Xinjiang facilitated the identification of gene families 
conserved among apicomplexan parasites [3-8]. In silico and experimental data demonstrated that CCp2 and CCp3 are well conserved among arthropod-borne apicomplexan parasites including Plasmodium, Theileria and Babesia and are consistently expressed in in vitro induced sexual stages $[9,10]$. Transcription of tick stagespecific genes, and associated protein expression, of $B$. bigemina has recently been documented during the parasite's development in the vector [11]. Bohaliga et al. [11] identified BBBOND_0204030 as a gene uniquely transcribed by $B$. bigemina tick stages and by in vitro induced $B$. bigemina sexual stages. The $B$. bigemina HAP2 protein, that is associated with the development of gametes in apicomplexan parasites [12], was found to be expressed by parasite stages developing in the midgut of infected ticks at day 3 post-repletion of female $R$. microplus ticks. In addition, studies identified and demonstrated protein expression of $B$. bigemina CCp1-3, a gene family also related to the development of sexual stages in apicomplexans [10], by B. bigemina in vitro induced sexual stages but not blood stages $[10,11]$. Finally, Johnson et al. [13] identified kinete stage-specific genes that are highly transcribed by $B$. bovis and $B$. bigemina kinetes as compared to blood stages. A B. bovis protein identified as BBOV_I002220 was specifically expressed by kinetes and is homologous to the protein encoded by B. bigemina BBBOND_0206730 gene (hereby renamed as BbiKSP, $B$. bigemina kinete stage-specific protein) [13]. In this study, we report for the first time the unique expression of BbiKSP by B. bigemina kinetes. In addition, we demonstrate that $\mathrm{CCp} 2$ and $\mathrm{CCp} 3$ proteins, previously shown to be expressed by in vitro induced sexual stages, are also expressed by kinetes isolated from the hemolymph of $B$. bigemina-infected adult female $R$. microplus ticks.

\section{Methods}

\section{In silico analysis}

Babesia bigemina tick specific genes were selected based on amino acid identity to $P$. falciparum, $P$. vivax and $B$. bovis [3-5]. The available annotated B. bigemina genome was used to determine features of selected genes [6]. The amino acid sequences of $P$. vivax Sal-1 strain CCp2 (GenBank: XP_001615829), P. falciparum 3D7 strain CCp3 (GenBank: XP_001348240), and B. bovis T2Bo strain BBOV_I002220 (GenBank: XP_001608872.1) were used to blast against the B. bigemina genome [6] using BLASTp software (https: //blast.ncbi.nlm.nih.gov/Blast). Clustal omega analysis (http://www.ebi.ac.uk/Tools/msa/ clustalo/) was used to determine the percent amino acid identity of proteins. The Simple Modular Architecture Research Tool (SMART) (http://smart.embl-heidelberg .de/) was used to identify predicted conserved domains. Presence of signal peptide, transmembrane domains and exterior protein presentation were evaluated using SignalP 4.1 server (http://www.cbs.dtu.dk/services/ SignalP/), TMHMM (http://www.cbs.dtu.dk/services/ TMHMM/) and PSIPRED (http://bioinf.cs.ucl.ac.uk/ psipred/), respectively.

\section{Isolation of B. bigemina kinetes from R. microplus adult female ticks}

To isolate B. bigemina kinetes from infected $R$. microplus adult female ticks, a naive splenectomized Holstein calf, four months of age and tested to be bovine babesiosis-free by nested PCR and competitive enzyme-linked immunosorbent assay $[14,15]$ was used for feeding an estimated 40,000 $R$. microplus larvae applied under a cloth back patch. After 14 days, when approximately $1 \%$ of the ticks had molted to adults, $10^{7} \mathrm{~B}$. bigemina Mexico strain infected erythrocytes were inoculated intravenously to synchronize tick acquisition feeding with an ascending $B$. bigemina parasitemia. Replete female ticks were collected at days 7 to 9 post-infection. Ticks were rinsed in tap water and placed into individual 24-well tissue culture plates and incubated at $26{ }^{\circ} \mathrm{C}$ in airtight containers with saturated $\mathrm{KNO}_{3}$ solution at $92 \%$ relative humidity for 6 days to allow accumulation of B. bigemina kinetes in the tick hemolymph. Ticks infected with $B$. bigemina were identified by removing the distal leg segment of engorged female ticks and exuding hemolymph onto slides. Slides were stained with Giemsa and examined by light microscopy for the presence of the parasite. Babesia bigemina kinetes were collected from infected female $R$. microplus ticks, as previous described [16]. In brief, ticks were placed on double-sided tape with the ventral side up. The cuticle was perforated using a 26 gauge needle and approximately $200 \mu$ of Hank's Balanced Salt Solution (HBSS) (Thermo Fisher Scientific, Waltham, MA, USA) injected into the membrane surrounding the base of the fourth leg coxal using a 33 gauge, 12.7 by $0.21 \mathrm{~mm}$ needle. Kinetes were collected by extraction of hemolymph-containing fluid with negative pressurized capillary tubing [16]. Hemolymph samples were pooled and the kinetes concentrated by centrifugation at $4000 \times g$ for $2 \mathrm{~min}$. Kinetes were used for RNA extraction and immunofluorescence assay (IFA) antigen.

\section{RNA isolation and CDNA synthesis}

Incubated replete female ticks were dissected daily from day 0 through day 6 post-incubation. Tick midgut and kinetes were collected in Trizol (Thermo Fisher Scientific) and stored at $-80{ }^{\circ} \mathrm{C}$. Total RNA isolation and DNase-Free treatment were performed following the manufacturer's protocol. One hundred ng of total RNA was utilized for cDNA synthesis using a Superscript III ${ }^{\mathrm{TM}}$ cDNA Synthesis Kit (Thermo Fisher Scientific) following 
the manufacturer's protocol. Synthesized cDNA was used in reverse transcription PCR (RT-PCR) to detect $B$. bigemina transcripts. Primer sets were designed based on $B$. bigemina genome sequences for BbiKSP (Forward 5'-GTG CAA AGC TGG TTG AAG AC-3' and Reverse 5'-GCA TGG ATA TCG TAC TGG TGT AG-3') and actin (BBBOND_0107357) (Forward 5'-ATC GCC GTT TAC ACT TCA CG-3' and Reverse 5'-GCC CCT TCC TCC TCG TAA TC-3'). Rhipicephalus microplus $\alpha$-tubulin primers (Forward 5'-CGT GCC GTA TTT GTT GAT C-3' and Forward 5'-AGA TTA GCT GCT CCG GGT G-3') were used as previously described [17]. The predicted amplicon sizes are $493 \mathrm{bp}, 131 \mathrm{bp}$ and $91 \mathrm{bp}$, respectively. RT-PCR reactions were conducted in $20 \mu \mathrm{l}$ containing $2 \mu \mathrm{l}$ of synthesized cDNA, $1 \mu \mathrm{l}$ of $10 \mu \mathrm{M}$ of each primer, $6 \mu \mathrm{l}$ of nuclease-free water and $10 \mu \mathrm{l}$ of RedTaq (Sigma-Aldrich, Saint Louis, MO, USA). The amplification conditions consisted of 3 min denaturation at $95{ }^{\circ} \mathrm{C}, 35$ repeated cycles of $30 \mathrm{~s}$ denaturation at $95^{\circ} \mathrm{C}$, $30 \mathrm{~s}$ annealing at $55^{\circ} \mathrm{C}$ and $30 \mathrm{~s}$ extension at $72{ }^{\circ} \mathrm{C}$, with a final 7 min extension at $72{ }^{\circ} \mathrm{C}$. Amplicons were resolved using $1 \%$ agarose gel electrophoresis. All PCR products were sequenced (Eurofins Genomics, Louisville, KY, USA).

\section{In vitro induction of $B$. bigemina sexual stages}

Babesia bigemina sexual stages were produced as previously described [11]. In brief, $B$. bigemina infected erythrocytes were grown in HL-1 medium (Lonza, Walkersville, MD) supplemented with $40 \%$ normal bovine serum, $10 \mathrm{mM}$ 3-[N-tris (hydroxymethyl) methylanino]2-hydroxypropanesulfonic acid (Sigma-Aldrich, St. Louis, MO, USA), and antibiotic/antimycotic (SigmaAldrich, St. Louis, MO, USA), pH 7.2, with a 5\% packed cell volume of bovine red blood cells. To increase parasitemia, cultures were incubated at $37{ }^{\circ} \mathrm{C}$ in $5 \% \mathrm{CO}_{2}$ and expanded without addition of erythrocytes [18]. When the parasitemia exceeded 10\%, B. bigemina sexual stages were induced with $20 \mathrm{mM}$ tris(2-carboxyethyl)phosphine (Thermo Fisher scientific) and incubated for $1 \mathrm{~h}$ at $37^{\circ} \mathrm{C}$ with $5 \% \mathrm{CO}_{2}$ as previously described [11]. Cultures were then washed once with fresh medium to remove excess reducing agent by centrifugation for $3 \mathrm{~min}$ at $2655 \times \mathrm{g}$. Parasites were cultured in fresh medium and incubated for up to $24 \mathrm{~h}$ at $37{ }^{\circ} \mathrm{C}$ with $5 \% \mathrm{CO}_{2}$. To analyze the formation of sexual stages, smears were made from all cultures at 6,15 and $24 \mathrm{~h}$ post-induction and stained with Hema 3 stain (Thermo Fisher Scientific). Cultures were collected at $0,6,15$ and $24 \mathrm{~h}$ post-induction for RNA extraction and IFA antigens. Total RNA isolation, DNasefree treatment and cDNA synthesis from non-induced and in vitro induced cultures at 6,15 and $24 \mathrm{~h}$ were performed as described above. cDNA at different time points were used in RT-PCR to detect the transcription of the genes of interest.

\section{Production of bovine antiserum}

To characterize $B$. bigemina protein expression by kinetes isolated from the hemolymph of $R$. microplus adult female ticks, rabbit antisera were raised against $\mathrm{CC} 2$ and CCp3 as previously described [10] and bovine antiserum was raised for BbiKSP. To generate bovine antisera against BbiKSP, synthetic peptides ranging from 13 to 20 amino acids were selected based on a proprietary algorithm identifying surface exposed B cell epitopes (BioSynthesis, Inc. Lewisville, TX, USA) using the sequence of B. bigemina BbiKSP. Three peptides were chosen for immunization: peptide1-KNQKTAIQDQRKDVDAKSKT (aa 117-136); peptide2-CQRHMPTERRDTN (aa 290-302) and peptide3-ALKPEETEDSGKES (aa 580593). A single spleen intact naive calf was subcutaneously immunized with individual preparations of each peptide in different draining lymph nodes. Each inoculation contained $50 \mu \mathrm{g}$ of a peptide conjugated to KLH plus $750 \mu \mathrm{g}$ of Quil-A Saponin adjuvant (Invivogen, San Diego, CA, USA). The calf received four booster inoculations at 3 week intervals. Before the first immunization and three weeks after the final immunization, serum was collected. For control sera, a second calf was vaccinated with KLH in Quil A Saponin using the same protocol. Animals were euthanized based on the euthanasia protocol approved by the Institutional Animal Care and Use Committee (IACUC).

\section{Immunofluorescence assays}

Fixed IFA were performed as previously described [10, $11,13]$. In brief, non-induced and in vitro induced $B$. bigemina cultures were collected at $0,6,15$, and $24 \mathrm{~h}$ and pelleted at $3000 \times g$ for $15 \mathrm{~min}$ at $4{ }^{\circ} \mathrm{C}$. Cells were washed two times with $1 \times$ PBS at $3000 \times g$ for $15 \mathrm{~min}$ at $4{ }^{\circ} \mathrm{C}$ and pellets suspended in PBS containing 3\% bovine serum albumin (BSA). These cells were used to make smears on glass microscope slides. Babesia bigemina kinetes were collected as described above and washed once with $1 \times$ PBS at $4000 \times g$ for $2 \mathrm{~min}$ at $4{ }^{\circ} \mathrm{C}$. The pellets were suspended in $1 \times$ PBS containing 3\% BSA and deposited into individual wells of Teflon coated slides and air dried. Slides were fixed in cold acetone for $1 \mathrm{~min}$ and blocked with $1 \times$ PBS containing $10 \%$ goat normal serum (PBSNGS) and incubated for $30 \mathrm{~min}$ at $37{ }^{\circ} \mathrm{C}$ in a humidity chamber. Slides were rinsed with $\mathrm{ddH}_{2} \mathrm{O}$ and air dried. Rabbit anti CCp2, anti-CCp3 [10] and bovine antiBbiKSP or anti-KLH primary antisera were diluted 1:20 in PBS-NGS and applied to individual wells. Slides were placed in a humidity chamber for $30 \mathrm{~min}$ at $37^{\circ} \mathrm{C}$. Then slides were rinsed once with $\mathrm{ddH}_{2} \mathrm{O}$ and washed three 
times for $10 \mathrm{~min}$ with cold $1 \times \mathrm{PBS}$. The slides were incubated at $37^{\circ} \mathrm{C}$ for $30 \mathrm{~min}$ with either goat anti-rabbit IgGAlexa Fluor 647 (Thermo Fisher Scientific) conjugated secondary antibody diluted 1:1000 in PBS-NGS for reactions to CCp2-3 antigen or goat anti-bovine IgG-FITC (SeraCare, Gaithersburg, MD, USA) conjugated secondary antibody diluted 1:100 in PBS-NGS for reactions to BbiKSP or KLH. All samples were washed twice in $1 \times$ PBS, once with $\mathrm{dd}_{2} \mathrm{O}$ and air dried. Finally, one drop of ProLong Gold anti-fade reagent with DAPI (Invitrogen, Eugene, OR, USA) was added to each sample under a coverslip. Identically produced negative controls were performed using pre-immune rabbit, pre-immune bovine or bovine anti-KLH serum as the primary antibody. All samples were visualized under a Leica microscope (Buffalo Grove, IL, USA) using LAS-X software.

\section{Results}

\section{In silico analysis}

In this study we focused on three proteins termed CCp2 and $\mathrm{CCp} 3$, previously identified as vector-specific proteins in other apicomplexa [10] and BbiKSP (Table 1). Bioinformatic analysis was performed to determine predicted features of the selected B. bigemina proteins such as molecular weight, signal peptide, and transmembrane domains (Table 2). Using this approach, we demonstrated that BbiKSP has a signal peptide at cleavage site between position 17 and 18. BbiKSP did not have any other identifiable conserved or transmembrane domains. Blasting BbiKSP against Babesia, Theileria, and Plasmodium species suggests BbiKSP is exclusively conserved within transovarially transmitted sensu stricto Babesia species as homologs of this gene were not found in intrastadial or transstadial transmitted parasites, including Plasmodium or Theileria (Table 3 and Additional file 1: Figure S1).

\section{Pattern of transcription of BbiKSP between Babesia bigemina blood and tick midgut stages}

RT-PCR analysis demonstrated that BbiKSP is transcribed in both $B$. bigemina blood and tick stages. BbiKSP transcripts were detected at various time-points in B. bigemina infected tick gut, as well as in kinetes from hemolymph (Fig. 1a). RT-PCR analysis for BbiKSP performed on tick midgut RNA samples displayed an on/ off pattern, with the day 0 signal providing results similar to blood stages. Days 1-2 midgut samples retained a B. bigemina actin signal but did not show a BbiKSP signal. Beginning at day 3 , all further midgut sampling dates were positive for BbiKSP. The specificity of Bbiactin primers was confirmed using cDNA made from $B$.

Table 1 Accession numbers of B. bigemina selected proteins and their homologous proteins in other arthropod-borne pathogens

\begin{tabular}{llllllllll}
\hline Protein name & B.big & B.bov & B.ova & B.sp.Xin & B.m & T.a & T.e & T.p & Pl \\
\hline BbiKSP & XP_012767701 & XP_001608872 & GBE61312 & ORM40592 & - & - & - & - & - \\
CCp2 & XP_012767423 & XP_001609893 & GBE61548 & ORM40789 & XP_012649113 & XP_952882 & XP_004830733 & XP_763844 & XP_001615829 \\
CCp3 & XP_012769523 & XP_001612018 & GBE58642 & ORM39994 & XP_021337760 & XP_953429 & XP_004831308 & XP_764433 & XP_001348240 \\
\hline
\end{tabular}

Abbreviations: B. big, Babesia bigemina; B. bov, Babesia bovis; B. ova, Babesia ovata; B. sp. Xin, Babesia sp. Xinjiang; B.m, Babesia microti; T.a, Theileria annulata; T.e, Theileria equi; T.p, Theileria parva; Pl, Plasmodium species

Table 2 Predicted features of the selected B. bigemina proteins

\begin{tabular}{lllllll}
\hline Protein & Chromosome & Exons & Signal peptide & Conserved domain & Nucleotide/Amino acid & MW (KDa) \\
\hline BbiKSP & 2 & 1 & Yes & None & $1839 / 612$ & 67.48 \\
CCp2 & 2 & 8 & No & LCCL & $4893 / 1630$ & 178.74 \\
CCp3 & 3 & 16 & Yes & LCCL & $3798 / 1265$ & 137.20 \\
\hline
\end{tabular}

Table 3 B. bigemina selected proteins and protein identities to other arthropod-borne pathogens

\begin{tabular}{|c|c|c|c|c|c|c|c|c|c|}
\hline \multirow{2}{*}{$\begin{array}{l}\text { B. bigemina } \\
\text { protein name }\end{array}$} & \multirow[t]{2}{*}{ Accession number } & \multicolumn{8}{|c|}{$\%$ amino acid identity } \\
\hline & & B.bov & B. ova & B.sp.Xin & $B m$ & $\mathrm{Ta}$ & $\mathrm{Te}$ & $T p$ & $P l$ \\
\hline BbiKSP & XP_012767701 & 22.94 & 65.47 & 28.50 & - & - & - & - & - \\
\hline CCp2 & XP_012767423 & 62.78 & 91.30 & 70.65 & 26.71 & 42.51 & 45.77 & 42.52 & 32.03 \\
\hline CCp3 & XP_012769523 & 72.85 & 92.60 & 80.78 & 31.29 & 52.70 & 52.72 & 53.00 & 37.03 \\
\hline
\end{tabular}


a

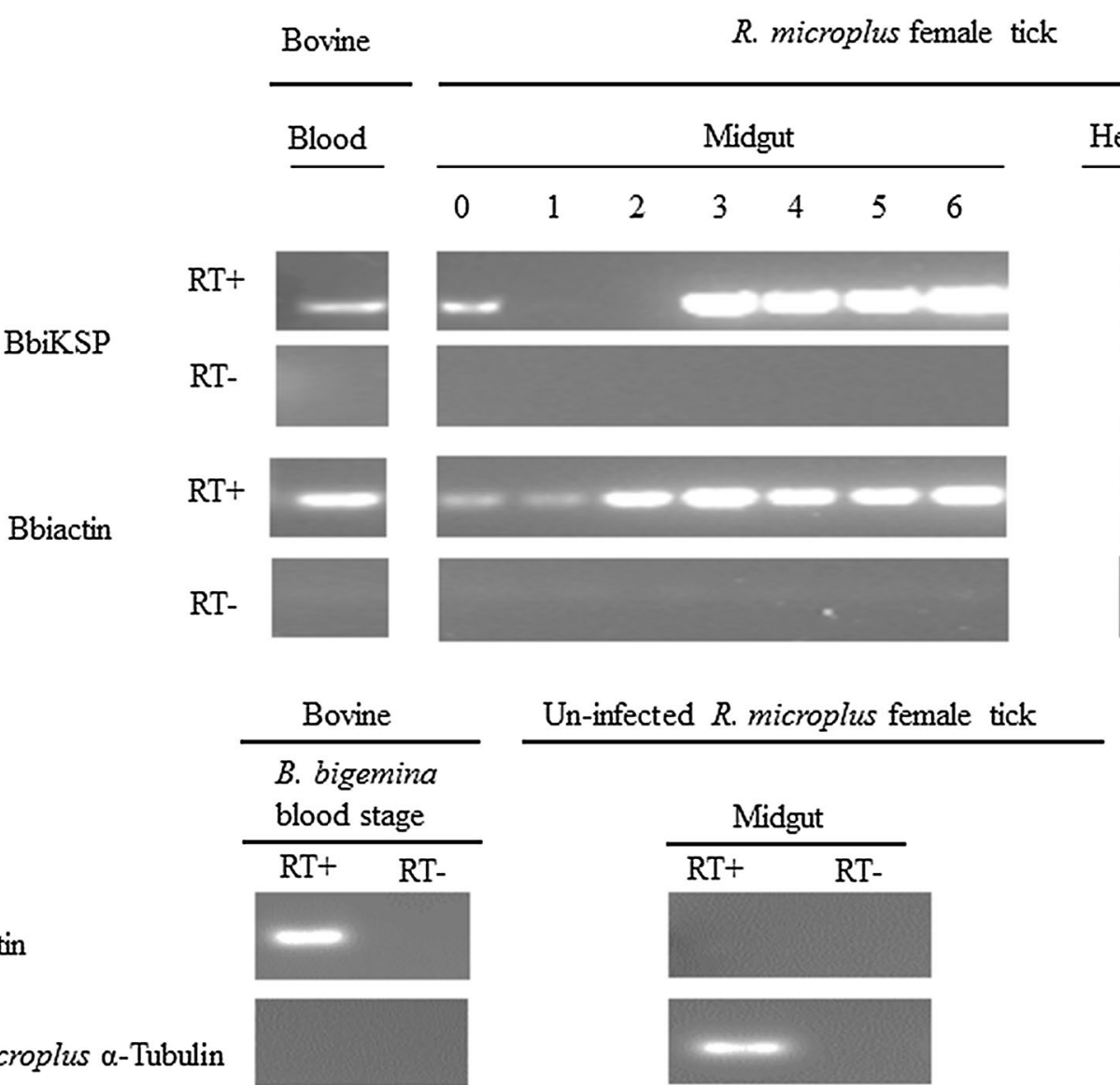

Fig. 1 Detection of BbiKSP transcripts during B. bigemina development in the mammalian and tick hosts. (a) Blood, midguts from day 0 to 6 post-incubation of engorged female ticks and infected hemolymph. To demonstrate that Bbiactin was specific for B. bigemina (b) B. bigemina actin was detected in infected blood but not in midgut from uninfected female tick. R. microplus a-Tubulin was used to demonstrate tick RNA quality. RT - and RT+ indicate the absence or presence of reverse transcriptase

bigemina-infected blood and uninfected midgut female tick RNA (Fig. 1b). The quality and integrity of tick cDNA was confirmed by RT-PCR amplification of $R$. microplus $\alpha$-Tubulin (Fig. 1b). RT(-) controls for all tissues were performed and did not yield any detectable product indicating the lack of genomic DNA in the samples. In addition, BbiKSP mRNA was transcribed by non-induced and in vitro induced cultures of B. bigemina at 15 and $24 \mathrm{~h}$ of post-induction but not $6 \mathrm{~h}$ post-induction (Fig. 2). These in vitro induction results were fully consistent with the ex vivo observation described above. All RT-PCR products obtained in these experiments were sequenced, and the amplicon identities confirmed.

\section{Analysis of protein expression by B. bigemina kinetes}

Protein expression of BbiKSP was only detected by IFA in B. bigemina kinetes and not in blood stages or in vitro induced sexual stages (Fig. 3a). Control reactions using antibodies against the peptide carrier KLH were negative for both in vitro induced sexual stages and kinetes, suggesting that the antibodies react specifically with the target proteins (Fig. 3d). For in vitro induced sexual stages, anti-CCp2 and $\mathrm{CCp} 3$ antibodies were used as positive controls for the IFA (Fig. 3b, c). However, CCp2 and CCp3 expression was not limited to sexual stages as a strong IFA reaction for both proteins to kinetes was observed (Fig. 3b, c). Blood stages did not express either CCp protein (Fig. $3 \mathrm{~b}, \mathrm{c}$ ). The reactions to all proteins did not appear to be confined to a particular region or organelle within the parasite, instead the reactions were uniform across the entire parasite. Table 4 summarizes the expression profiles of BbiKSP, CCp2 and CCp3.

\section{Discussion}

Babesia bigemina has a complex life-cycle that includes asexual reproduction in the bovine host and sexual reproduction in tick vectors $[1,2]$. When adult female ticks feed on an animal infected with B. bigemina, the 


\begin{tabular}{|c|c|c|c|c|c|c|c|c|}
\hline & & & & cult & & & & \\
\hline & & & & & & & & \\
\hline & RT- & $\mathrm{RT}+$ & & RT- & & & RT+ & \\
\hline & $\mathrm{Oh}$ & Oh & $6 \mathrm{~h}$ & $15 \mathrm{~h}$ & $24 \mathrm{~h}$ & $6 \mathrm{~h}$ & $15 \mathrm{~h}$ & $24 \mathrm{~h}$ \\
\hline BbiKSP & & & & & & & & \\
\hline Bbiactin & & & & & & & & \\
\hline
\end{tabular}

Fig. 2 Detection of BbiKSP transcripts in non-induced blood and in vitro induced cultures at multiples time points. Bbiactin was used as a reference gene. RT - and RT+ indicate the absence or presence of reverse transcriptase
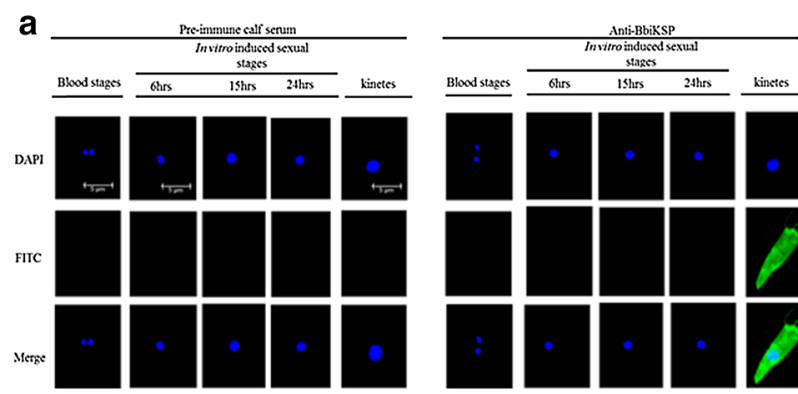

b

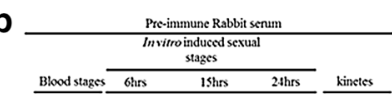

$$
\text { DAPI }
$$

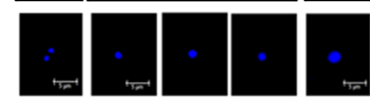

AF 677
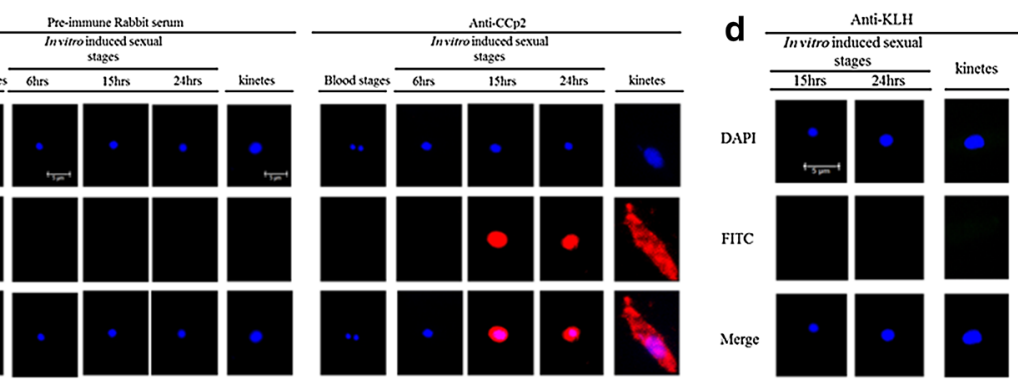

Fig. 3 Immunofluorescence assays demonstrating expression of BbiKSP (a), CCp 2 (b), CCp3 (c) by B. bigemina kinetes but not blood stages and bovine anti-KLH as control (d). Scale-bars: 3 and $5 \mu \mathrm{m}$

Table 4 Summary of expression profiles of BbiKSP, CCp2 and CCp3

\begin{tabular}{|c|c|c|c|c|c|}
\hline \multirow[t]{2}{*}{ Expression profile } & \multirow[t]{2}{*}{ Blood stages } & \multicolumn{3}{|c|}{ Induced sexual stages } & \multirow[t]{2}{*}{ Kinetes } \\
\hline & & $6 \mathrm{~h}$ & $15 \mathrm{~h}$ & $24 \mathrm{~h}$ & \\
\hline \multicolumn{6}{|l|}{ BbiKSP } \\
\hline mRNA & + & - & + & + & + \\
\hline Protein & - & - & - & - & + \\
\hline \multicolumn{6}{|l|}{ CCp2 } \\
\hline mRNA & + & + & + & + & + \\
\hline Protein & - & - & + & + & + \\
\hline \multicolumn{6}{|l|}{ CCp3 } \\
\hline mRNA & + & + & + & + & + \\
\hline Protein & - & - & + & + & + \\
\hline
\end{tabular}

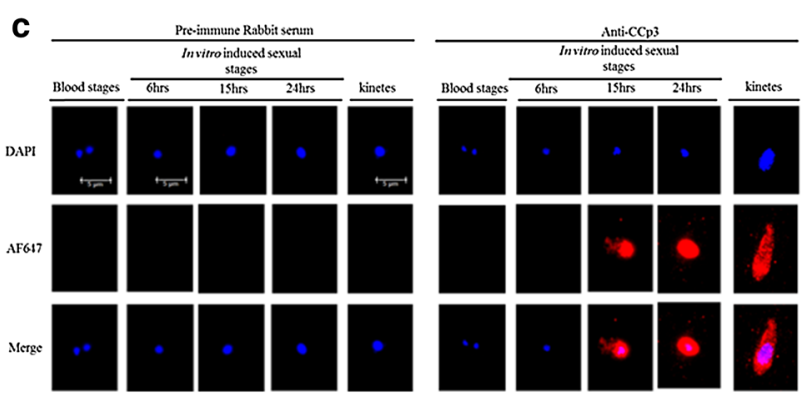

parasite sexually reproduces within the biological vector, undergoes several morphological changes, and interacts with multiple cell types and tissues including tick midgut, ovaries and salivary glands before transmission $[1,2]$. Proteins that are expressed by tick stages of $B$. bigemina remain uncharacterized.

In this study, we demonstrated that BbiKSP mRNA was transcribed in the stages of the parasite present in infected bovine blood and infected tick midgut and hemolymph. However, BbiKSP polypeptides were not detected in either blood stages or in vitro induced sexual stages, including $B$. bigemina gametocytes, gametes and zygotes. This is in agreement with a previous finding demonstrating expression of its $B$. bovis homologous 
protein BboKSP, BBOV_I002220, exclusively in B. bovis kinetes despite the presence of transcripts, albeit at low levels, in infected blood [13]. Our comparative in silico data demonstrated that BbiKSP was conserved among Babesia parasites including B. bovis, B. ovata and $B$. sp. Xinjiang (Additional file 1: Figure S1), with the greatest identity, $65.47 \%$, shared between B. bigemina and $B$. ovata. Previously, BboKSP, which contains a $C$ terminal GPI anchor, was characterized with cell surface staining techniques to suggest it was on the exterior surface of the kinete [13]. No homologs of BbiKSP were found in the genomes of the closely related Plasmodium and Theileria parasites, nor in Babesia (sensu lato) parasites such as $B$. microti. While these three parasites species lacking a KSP gene undergo sexual reproduction in their arthropod vectors, none are transovarially transmitted. In contrast, the data suggest that all transovarially transmitted Babesia (sensu stricto) species, such as B. bovis, contain genes that are homologous to BbiKSP $[7,8,13]$. It is possible that BbiKSP encodes for a protein involved in parasite invasion into the ovaries and, thus, is required for transovarial transmission.

In silico predictions of BbiKSP cellular localization by TMHMM and PSIPRED indicated that the protein is extracellular. This feature is compatible with the premise that the protein interacts with host moieties to invade the invertebrate host cells. The nature of this interaction is unknown, however, a possible mechanism that could facilitate transovarial transmission of the parasite might involve BbiKSP and vitellogenin. Kinetes develop from parasite zygotes that have invaded basophilic cells in the tick midgut. Basophilic cells are major producers of vitellogenin, a lipoglycoprotein that is used to form the yolks of developing eggs [19]. RNAi experiments interfering with vitellogenin receptors (VgR) have abolished transovarial transmission of B. bovis in R. microplus ticks [20]. The fixed IFA results for BbiKSP are consistent with an exterior membrane-associated location, however, confirmation of the extracellular display of BbiKSP awaits further testing using live, intact $B$. bigemina kinetes.

Additionally, we demonstrated that $\mathrm{CCp} 2$ and $\mathrm{CCp} 3$ were expressed by B. bigemina kinetes. Members of the CCp family have been previously identified and characterized by related apicomplexan parasites including Plasmodium, Theileria and Babesia $[9,10,21]$. The presence of $\mathrm{CCp}$ orthologous proteins in related apicomplexan parasites suggests potentially conserved functions for this family. Our data suggest that B. bigemina $\mathrm{CCp} 2$ and $\mathrm{CCp} 3$ proteins may also play a role in $B$. bigemina transovarial transmission.

A surprising finding in this study is the presence of RNA transcript without translated polypeptide. This has been described before for B. bigemina CCp 1-3 [10] and, as mentioned previously, for BboKSP [13]. Interestingly, a similar lack of correlation among RNA and protein levels was previously reported for the members of the RAP-1 gene family in B. bigemina and other rhoptry proteins expressed by Plasmodium parasites. It was postulated that these findings may reflect a gene expression regulatory mechanism used by the parasite to adapt to variations in life- cycle, such as the different vertebrate and invertebrate vector environments [22]. Gene expression is controlled at many different stages and by many different mechanisms, thus, establishing correlations between mRNA and protein levels are not always possible. There are cases where transcript copy number strongly correlates with protein levels; however, regulation of gene expression may be controlled upstream of transcription and/or translation, resulting in poor correlation between mRNA and protein levels. Post-transcriptional modifications of mRNA and the activity of microRNAs or other regulatory molecules may be the reasons for low or undetectable protein expression despite having corresponding high mRNA levels in a cell [23]. Some of these regulatory mechanisms may be operating as regulons for the synthesis of proteins in Babesia parasites, but unfortunately, these regulatory mechanisms remain unexplored in most apicomplexan parasites and at this point we can only speculate on the possible explanations.

Regardless of the translational control mechanism involved, it is interesting to note that mRNA for BbiKSP was not detected in midguts from days 1-2 of replete, incubated females before returning to detectable levels on day 3. The pattern of BbiKSP transcription indicates significant changes in transcriptional regulation for this gene during the early development of the parasite in the vector. This is consistent with our induced sexual stage results that showed an initial loss of BbiKSP transcription signal at $6 \mathrm{~h}$ that subsequently returned at $15 \mathrm{~h}$. We postulated that sexual stages developing in tick midgut undergo a similar change in transcription patterns. This is supported by our previous work that demonstrated a five log increase in transcript for BbiKSP in kinetes as compared to blood stages [13]. Apicomplexa use a suite of transcription control factors known as AP2 that differentially regulate gene expression. Specific AP2, such as the described AP2-G in Plasmodium gametes, direct gene expression in a life-cycle stage dependent fashion [24]. A recent review showed that Babesia also have AP2 and that they possess a homolog for AP2-G [25]. The return of BbiKSP transcripts by day 3 in replete females with concomitant polypeptide expression by circulating kinetes suggests a turnover in which AP2 are directing gene transcription. This may also include genes other than BbiKSP that ensure the complete translation of the mRNA and appropriate cellular display. The 
stage-specific expression of BbiKSP appears to be similar to 6-Cys-C that is restricted to the kinete stage of $B$. bovis [26]. In contrast, polypeptide expression of genes important during B. bigemina blood stages, such as Rhoptry Associated Protein-1a (RAP-1a), can be detected by IFA throughout the entire life-cycle of the parasite. However, RAP-1a does not appear to localize to a rhoptry organelle in kinetes but, rather, is distributed in a speckled pattern within the parasite [27]. This resembles the inappropriate cellular partitioning that occurs with ROP2 in the absence of adaptor complex 1 in Toxoplasma gondii [23], reinforcing the suggestion that secondary signals are required by apicomplexans for complete translation and display of surface proteins. It is also possible that expression of the Babesia KSPs described hereby are also subjected to such finely regulated mechanisms, resulting in the expression of KSP proteins only when and where they are required for a function that is related to the invasion of ovary tissues of the tick, in order to allow occurrence of the transovarial transmission mechanisms typical of Babesia (sensu stricto) parasites.

\section{Conclusions}

Babesia bigemina is the one of most important parasites responsible for bovine babesiosis that results in a high negative economic impact on the cattle industry. To improve bovine babesiosis control, understanding the events of parasite development within the vector and characterizing parasite proteins expressed by parasite tick stages is crucial. In this study, we document BbiKSP expression as unique to the kinete stage. In addition, CCp2 and CCp3 proteins were found to be expressed by kinetes. The tick stage-specific expression of these parasite proteins, and their possible involvement in the mechanism of transovarial transmission, strongly suggest their future use as potential candidates for developing Babesia transmission blocking vaccines. Further investigations are needed to determine the role and function of these proteins during the parasite development within adult female ticks.

\section{Additional file}

Additional file 1: Figure S1. Amino acids alignment of BbiKSP among Babesia species with their accession numbers: B. bigemina, XP_012767701.1; B. bovis, XP_001608872.1; B. ovata, GBE61312.1; B. sp. Xinjiang, ORM40592.1.

\section{Abbreviation}

PBS: phosphate-buffered saline.

\section{Acknowledgments}

We thank Ralph Horn, James Allison and Kathy Mason of the U.S. Department of Agriculture, Animal Disease Research Unit, for their excellent animal care.
We appreciate the excellent assistance of Paul Lacy and Jacob Laughery in maintaining $B$. bigemina cultures.

\section{Authors' contributions}

All authors contributed to the critical review of the final manuscript. GARB, WCJ, NST, HEH and MWU conceived and designed the study, and critically revised the paper. GARB performed the experiments. GARB, WCJ and MWU performed the data analysis. GARB, WCJ, HEH, NST, CES, RGB, GAS and MWU drafted the manuscript. All authors read and approved the final manuscript.

\section{Funding}

This project was supported by USDA-ARS CRIS project number 2090-32000039-00D. GARB was supported by the Libyan Ministry of Higher Education and Scientific Research.

\section{Availability of data and materials}

The data supporting the conclusions of this article are included within the article and its additional files.

\section{Ethics approval and consent to participate}

This study was approved by the Institutional Animal Care and Use Committee of the University of Idaho, Moscow, Idaho, in accordance with institutional guidelines based on the U.S. National Institutes of Health Guide for the Care and Use of Laboratory Animals.

\section{Consent for publication}

Not applicable.

\section{Competing interests}

The authors declare that they have no competing interests.

\section{Author details}

${ }^{1}$ Program in Vector-borne Diseases, Department of Veterinary Microbiology and Pathology, Washington State University, Pullman, WA 99164, USA. 2 Animal Disease Research Unit, USDA-ARS, Pullman, WA 99164-6630, USA.

${ }^{3}$ Department of Entomology, Faculty of Science, Cairo University, Giza 12613, Egypt. ${ }^{4}$ The Paul G. Allen School for Global Animal Health, Washington State University, Pullman, WA 99164-70403, USA.

Received: 31 January 2019 Accepted: 23 May 2019

Published online: 28 May 2019

\section{References}

1. Bock R, Jackson L, de Vos A, Jorgensen W. Babesiosis of cattle. Parasitology. 2004;129(Suppl.):S247-69.

2. Riek RF. The life cycle of Babesia bigemina (Smith \& Kilborne, 1893) in the tick vector Boophilus microplus (Canestrini). Aust J Agric Res. 1964;15:802-21.

3. Gardner MJ, Hall N, Fung E, White O, Berriman M, Hyman RW, et al. Genome sequence of the human malaria parasite Plasmodium falciparum. Nature. 2002:419:498-511.

4. Bright AT, Tewhey R, Abeles S, Chuquiyauri R, Llanos-Cuentas A, Ferreira $\mathrm{MU}$, et al. Whole genome sequencing analysis of Plasmodium vivax using whole genome capture. BMC Genom. 2012;13:262.

5. Brayton KA, Lau AO, Herndon DR, Hannick L, Kappmeyer LS, Berens SJ, et al. Genome sequence of Babesia bovis and comparative analysis of apicomplexan hemoprotozoa. PLoS Pathog. 2007;3:1401-13.

6. Jackson AP, Otto TD, Darby A, Ramaprasad A, Xia D, Echaide IE, et al. The evolutionary dynamics of variant antigen genes in Babesia reveal a history of genomic innovation underlying host-parasite interaction. Nucleic Acids Res. 2014;42:7113-31.

7. Yamagishi J, Asada M, Hakimi H, Tanaka TQ, Sugimoto C, Kawazu S-I. Whole-genome assembly of Babesia ovata and comparative genomics between closely related pathogens. BMC Genom. 2017;18:832.

8. Niu Q, Liu Z, Yang J, Gao S, Pan Y, Guan G, et al. Genetic characterization and molecular survey of Babesia sp. Xinjiang infection in small ruminants and ixodid ticks in China. Infect Genet Evol. 2017;49:330-5.

9. Pradel G, Hayton K, Aravind L, lyer LM, Abrahamsen MS, Bonawitz A, et al. A multidomain adhesion protein family expressed in Plasmodium 
falciparum is essential for transmission to the mosquito. J Exp Med. 2004;199:1533-44.

10. Bastos RG, Suarez CE, Laughery JM, Johnson WC, Ueti MW, Knowles DP. Differential expression of three members of the multidomain adhesion CCp family in Babesia bigemina, Babesia bovis and Theileria equi. PLOS One. 2013;8:e67765.

11. Bohaliga GAR, Johnson WC, Taus NS, Hussein HE, Bastos RG, Suarez $\mathrm{CE}$, et al. Identification of a putative methyltransferase gene of Babesia bigemina as a novel molecular biomarker uniquely expressed in parasite tick stages. Parasit Vectors. 2018;11:480.

12. Camacho-Nuez M, Hernández-Silva DJ, Castañeda-Ortiz EJ, ParedesMartínez ME, Rocha-Martínez MK, Alvarez-Sánchez ME, et al. Hap2, a novel gene in Babesia bigemina is expressed in tick stages, and specific antibodies block zygote formation. Parasit Vectors. 2017;10:568.

13. Johnson WC, Taus NS, Reif KE, Bohaliga GA, Kappmeyer LS, Ueti MW. Analysis of stage-specific protein expression during Babesia bovis development within female Rhipicephalus microplus. J Proteome Res. 2017;16:1327-38

14. Suarez CE, Laughery JM, Schneider DA, Sondgeroth KS, McElwain TF. Acute and persistent infection by a transfected Mo7 strain of Babesia bovis. Mol Biochem Parasitol. 2012;185:52-7.

15. Goff WL, Molloy JB, Johnson WC, Suarez CE, Pino I, Rhalem A, et al. Validation of a competitive enzyme-linked immunosorbent assay for detection of antibodies against Babesia bovis. Clin Vaccine Immunol. 2006;13:1212-6.

16. Howell JM, Ueti MW, Palmer GH, Scoles GA, Knowles DP. Transovarial transmission efficiency of Babesia bovis tick stages acquired by Rhipicephalus (Boophilus) microplus during acute infection. J Clin Microbiol. 2007:45:426-31.

17. Ueti MW, Palmer GH, Kappmeyer LS, Scoles GA, Knowles DP. Expression of equi merozoite antigen 2 during development of Babesia equi in the midgut and salivary gland of the vector tick Boophilus microplus. J Clin Microbiol. 2003:41:5803-9.

18. Goff WL, Yunker CE. Babesia bovis: increased percentage parasitized erythrocytes in cultures and assessment of growth by incorporation of [3H]hypoxanthine. Exp Parasitol. 1986;62:202-10.

19. Xavier MA, Tirloni L, Pinto AFM, Diedrich JK, Yates JR 3rd, Mulenga A, et al. A proteomic insight into vitellogenesis during tick ovary maturation. Sci Rep. 2018;8:4698.
20. Hussein HE, Johnson WC, Taus NS, Suarez CE, Scoles GA, Ueti MW. Silencing expression of the Rhipicephalus microplus vitellogenin receptor gene blocks Babesia bovis transmission and interferes with oocyte maturation. Parasit Vectors. 2019;12:7.

21. Becker CAM, Malandrin L, Depoix D, Larcher T, David PH, Chauvin A Bischoff E, Bonnet S. Identification of three CCp genes in Babesia divergens: novel markers for sexual stages parasites. Mol Biochem Parasitol. 2010;174:36-43.

22. Suarez $\mathrm{CE}$, Palmer $\mathrm{GH}$, Florin-Christensen $\mathrm{M}$, Hines SA, Hötzel I, McElwain TF. Organization, transcription, and expression of rhoptry associated protein genes in the Babesia bigemina rap-1 locus. Mol Biochem Parasitol. 2003:127:101-12.

23. Ngo HM, Yang M, Paprotka K, Pypaert M, Hoppe H, Joiner KA. AP-1 in Toxoplasma gondii mediates biogenesis of the rhoptry secretory organelle from a post-golgi compartment. J Biol Chem. 2003;278:5343-52.

24. Sinha A, Hughes KR, Modrzynska KK, Otto TD, Pfander C, Dickens NJ, et al. A cascade of DNA-binding proteins for sexual commitment and development in Plasmodium. Nature. 2014;507:253-7.

25. Alzan HF, Knowles DP, Suarez CE. Comparative bioinformatics analysis of transcription factor genes indicates conservation of key regulatory domains among Babesia bovis, Babesia microti, and Theileria equi. PLoS Neglect Trop Dis. 2016;10:e0004983.

26. Alzan HF, Lau AOT, Knowles DP, Herndon DR, Ueti MW, Scoles GA, et al. Expression of 6-Cys gene superfamily defines Babesia bovis sexual stage development within Rhipicephalus microplus. PLoS ONE. 2016;11:e0163791.

27. Mosqueda J, Falcon A, Antonio Alvarez J, Alberto Ramos J, OropezaHernandez LF, Figueroa JV. Babesia bigemina sexual stages are induced in vitro and are specifically recognized by antibodies in the midgut of infected Boophilus microplus ticks. Int J Parasitol. 2004;34:1229-36.

\section{Publisher's Note}

Springer Nature remains neutral with regard to jurisdictional claims in published maps and institutional affiliations.
Ready to submit your research? Choose BMC and benefit from:

- fast, convenient online submission

- thorough peer review by experienced researchers in your field

- rapid publication on acceptance

- support for research data, including large and complex data types

- gold Open Access which fosters wider collaboration and increased citations

- maximum visibility for your research: over $100 \mathrm{M}$ website views per year

At BMC, research is always in progress.

Learn more biomedcentral.com/submissions 